\title{
Tachyon inflation in an AdS braneworld with back-reaction
}

\author{
Neven Bilić ${ }^{1 *}$ Dragoljub Dimitrijevic ${ }^{2 \dagger}$, Goran Djordjevic ${ }^{2 \ddagger}$, and Milan Milosevićc ${ }^{2 \S}$ \\ ${ }^{1}$ Division of Theoretical Physics, Rudjer Bošković Institute, Zagreb, Croatia \\ ${ }^{2}$ Department of Physics, University of Niš, Srbija
}

August 8, 2018

\begin{abstract}
We analyze the inflationary scenario based on the tachyon field coupled with the radion of the second Randall-Sundrum model (RSII). The tachyon Lagrangian is derived from the dynamics of a 3-brane moving in the five dimensional bulk. The $\mathrm{AdS}_{5}$ geometry of the bulk is extended to include the radion. Using the Hamiltonian formalism we find four nonlinear field equations supplemented by the modified Friedmann equations of the RSII braneworld cosmology. After a suitable rescaling we reduce the parameters of our model to only one free parameter related to the brane tension and the $\mathrm{AdS}_{5}$ curvature. We solve the equations numerically assuming a reasonably wide range of initial conditions determined by physical considerations. Varying the free parameter and initial conditions we confront our results with the Planck 2015 data.
\end{abstract}

\section{Introduction}

The inflationary universe scenario [1, 2, 3] in which the early universe undergoes a rapid expansion has been generally accepted as a solution to the horizon problem and some other related problems of the standard big-bang cosmology. The origin of the field that drives inflation is still unknown and is subject to speculations. Among many models of inflation a popular class comprise tachyon inflation models [4, 5, 6, 7, 8, 9, 10, 11, 12, 13, 14. These models are of particular interest as in these models inflation is driven by the tachyon field originating in string theory.

The tachyon potential is derived from string theory and has to satisfy some definite properties to describe tachyon condensation and other requirements in string theory. However, Kofman and Linde have shown [7] that the slow-roll conditions are not compatible with a string coupling much smaller than one, and the compactification length scale much larger

\footnotetext{
*bilic@irb.hr

†ddrag@pmf.ni.ac.rs

${ }_{\ddagger}^{\ddagger}$ gorandj@junis.ni.ac.rs

§mmilan@seenet-mtp.info
} 
than the Planck length. This leads to the density fluctuations produced during inflation being incompatible with observational constraint on the amplitude of the scalar perturbations. This criticism is based on the string theory motivated values of the parameters in the tachyon potential, i.e., the brane tension and the parameters in the four-dimensional Newton constant obtained via conventional string compactification. Of course, if one relaxes the string theory constraints on the above mentioned parameters, the effective tachyon theory will naturally lead to a type of inflation which will slightly deviate from the conventional inflation based on the canonical scalar field theory. Steer and Vernizzi [11] have noted a deviation from the standard single field inflation in the second order consistency relations. Based on their analysis they concluded that the tachyon inflation could not be ruled out by the then available observations. It seems like the present observations [15] could perhaps discriminate between different tachyon models and disfavor or rule out some of these models (for a recent discussion on phenomenological constraints imposed by Planck 2015, see, e.g., ref [16]).

A simple tachyon model can be analyzed in the framework of the second Randall-Sundrum (RSII) model [17]. The RSII model was originally proposed as a possible mechanism for localizing gravity on the $3+1$ universe embedded in a $4+1$ dimensional spacetime without compactification of the extra dimension. The model is a $4+1$ dimensional Anti de Sitter $\left(\mathrm{AdS}_{5}\right)$ universe containing two 3 -branes with opposite tensions separated in the fifth dimension: observers reside on the positive tension brane and the negative tension brane is pushed off to infinity. The Planck mass scale is determined by the curvature of the AdS spacetime rather then by the size of the fifth dimension.

The fluctuation of the interbrane distance along the extra dimension implies the existence of the so called radion - a massless scalar field that causes a distortion of the bulk geometry. In this regard, a stabilization mechanism of the interbrane distance has been proposed [18] by assuming the presence of scalar fields in the bulk. The stabilization mechanism is relevant for the RSI model where the interbrane distance is kept finite. In RSII model, as the negative tension brane is pushed off to infinity the radion disappears. However, it has been shown by Kim, Tupper, and Viollier [19] that a disappearance of the radion in RSII is an artifact of linear theory and hence, when going beyond linear theory the radion remains a dynamical field in the RSII model. Moreover, owing to the radion, the distance between branes remains finite in the RSII limit of infinite coordinate bulk even though the coordinate position of the second brane is infinite.

The presence of the radion may have interesting physical implications. The radion field has been proposed as an inflaton [20]. It has been shown [21, 22] that the interaction of the radion with the tachyon can alter the tachyon equation of state: by averaging over large scales the effective equation of state describes the warm dark matter. One of our aims here is to answer the question whether radion could drastically change the early cosmology in the framework of the RSII braneworld model.

In this paper we propose a simple tachyon condensate as a model for inflation and we analyze two effects: the coupling of the tachyon with the radion and the modification of the standard cosmology in the RSII scenario. To study these effects we will consider an additional dynamical 3-brane moving in the $\mathrm{AdS}_{5}$ background of the RSII model. The action of the $3+1$ dimensional brane in the five dimensional bulk is equivalent to the DiracBorn-Infeld (DBI) description of the Nambu-Goto 3-brane. [23, 24]. It is a simple matter to 
show that this additional 3-brane behaves effectively as a tachyon with the inverse quartic potential [22]. The tachyon model of this kind falls into the class of the power-law tachyon potentials $V(\theta) \propto \theta^{-n}$ with $n>2$ which drive a dark-matter attractor [25]: for $\theta \rightarrow \infty$, the pressure tends to unity very quickly with the unpleasant feature of cold dark matter (CDM) domination at the end of inflation. Actually, this problem is imminent for all tachyon models with the ground state at $\theta \rightarrow \infty$ [7]. The tachyon field rolls towards its ground state without oscillating about it and the conventional reheating mechanism does not work. Typically these scenarios suffer from a reheating problem, since gravitational particle production is not efficient compared to the standard non-gravitational particle production by an oscillating inflaton field. This will be discussed in more detail at the end of Sec. 3 .

The remainder of the paper is organized as follows. In Sec. 2 we present the RSII model with back-reaction. The system of dynamical equations is presented in a dimensionless form suitable for the calculation of the expansion rate and slow-roll parameters. In Sec. 3 the slow-roll approximation and initial conditions for our model are discussed in detail. Numerical results are presented and discussed in Sec. 4. In the concluding section, Sec. 5 , we summarize our results and give conclusions.

\section{Randall-Sundrum model with back-reaction}

The bulk spacetime of the extended RSII model which includes the back-reaction of the radion [19] in Fefferman-Graham coordinates is described by the line element

$$
d s_{(5)}^{2}=G_{a b} d X^{a} d X^{b}=\frac{1}{k^{2} z^{2}}\left[\left(1+k^{2} z^{2} \eta(x)\right) g^{\mu \nu} d x^{\mu} d x^{\nu}-\frac{1}{\left(1+k^{2} z^{2} \eta(x)\right)^{2}} d z^{2}\right],
$$

where $k=1 / \ell$ is the inverse of the AdS curvature radius $\ell$ and $\eta(x)$ is the radion field. The observer brane is placed at $z=\ell$ and $g_{\mu \nu}$ is the metric on the brane.

Consider a dynamical 3-brane moving in the bulk. The brane Lagrangian describes the dynamic of the tachyon field modified by the interaction with the radion. After integrating out the fifth coordinate the total effective action is given by [19]

$$
S=\int d^{4} x \sqrt{-g}\left(-\frac{R}{16 \pi G}+\frac{1}{2} g^{\mu \nu} \Phi_{, \mu} \Phi_{, \nu}\right)+S_{\mathrm{br}},
$$

where $\Phi$ is the canonically normalized radion field related to $\eta$ as

$$
\eta=\sinh ^{2}\left(\sqrt{\frac{4 \pi G}{3}} \Phi\right)
$$

The brane action $S_{\text {br }}$ is derived in Ref. [22] in terms of the induced metric or the "pull back" of the bulk space-time metric $G_{a b}$ to the brane,

$$
g_{\mu \nu}^{(\text {ind })}=G_{a b} \frac{\partial X^{a}}{\partial x^{\mu}} \frac{\partial X^{b}}{\partial x^{\nu}} .
$$

The action takes the form

$$
S_{\mathrm{br}}=-\sigma \int d^{4} x \sqrt{-\operatorname{det} g_{\mu \nu}^{\text {ind }}}=-\int d^{4} x \sqrt{-g} \frac{\sigma}{k^{4} \Theta^{4}}\left(1+k^{2} \Theta^{2} \eta\right)^{2} \sqrt{1-\frac{g^{\mu \nu} \Theta_{, \mu} \Theta_{, \nu}}{\left(1+k^{2} \Theta^{2} \eta\right)^{3}}},
$$


where $\sigma$ denotes the brane tension and $\Theta$ is the tachyon field.

In the absence of the radion $(\Phi=0)$ the brane action is just the tachyon condensate with the inverse quartic potential

$$
S_{\mathrm{br}}^{(0)}=-\int d^{4} x \sqrt{-g} \frac{\lambda}{\Theta^{4}} \sqrt{1-g^{\mu \nu} \Theta_{, \mu} \Theta_{, \nu}}
$$

where

$$
\lambda=\frac{\sigma}{k^{4}}
$$

The combined brane-radion Lagrangian reads

$$
\mathcal{L}=\frac{1}{2} g^{\mu \nu} \Phi_{, \mu} \Phi_{, \nu}-\frac{\lambda \psi^{2}}{\Theta^{4}} \sqrt{1-\frac{g^{\mu \nu} \Theta_{, \mu} \Theta_{, \nu}}{\psi^{3}}},
$$

where

$$
\psi=1+k^{2} \Theta^{2} \eta
$$

Hence, in our model, the tachyon is as usual the Dirac-Born-Infeld type scalar field whereas the radion is a canonical scalar. In the following we will assume the spatially flat FRW spacetime on the observer brane with four dimensional line element in the standard form

$$
d s^{2}=g_{\mu \nu} d x^{\mu} d x^{\nu}=d t^{2}-a^{2}(t)\left(d r^{2}+r^{2} d \Omega^{2}\right) .
$$

The treatment of our system in a cosmological context is conveniently performed in the Hamiltonian formalism. For this purpose we first define the conjugate momentum fields as

$$
\Pi_{\Phi}^{\mu}=\frac{\partial \mathcal{L}}{\partial \Phi_{, \mu}}, \quad \Pi_{\Theta}^{\mu}=\frac{\partial \mathcal{L}}{\partial \Theta_{, \mu}} .
$$

In the cosmological context $\Pi_{\Phi}^{\mu}$ and $\Pi_{\theta}^{\mu}$ are time-like so we may also define their magnitudes as

$$
\Pi_{\Phi}=\sqrt{g_{\mu \nu} \Pi_{\Phi}^{\mu} \Pi_{\Phi}^{\nu}}, \quad \Pi_{\theta}=\sqrt{g_{\mu \nu} \Pi_{\theta}^{\mu} \Pi_{\theta}^{\nu}} .
$$

The Hamiltonian density may be derived from the stress tensor corresponding to the Lagrangian (86) or by the Legendre transformation. Either way one finds [22]

$$
\mathcal{H}=\frac{1}{2} \Pi_{\Phi}^{2}+\frac{\lambda \psi^{2}}{\Theta^{4}} \sqrt{1+\Pi_{\Theta}^{2} \Theta^{8} /\left(\lambda^{2} \psi\right)} .
$$

For later use we may also need the Hamiltonian density in terms of $\Phi_{, \mu}$ and $\Theta_{, \mu}$,

$$
\mathcal{H}=\frac{1}{2} g^{\mu \nu} \Phi_{, \mu} \Phi_{, \nu}+\frac{\lambda \psi^{2}}{\Theta^{4}}\left(1-\frac{g^{\mu \nu} \Theta_{, \mu} \Theta_{, \nu}}{\psi^{3}}\right)^{-1 / 2} .
$$

Next, we can write Hamilton's equations in the form

$$
\begin{aligned}
\dot{\Phi} & =\frac{\partial \mathcal{H}}{\partial \Pi_{\Phi}}, \\
\dot{\Theta} & =\frac{\partial \mathcal{H}}{\partial \Pi_{\Theta}}, \\
\dot{\Pi}_{\Phi}+3 H \Pi_{\Phi} & =-\frac{\partial \mathcal{H}}{\partial \Phi}, \\
\dot{\Pi}_{\Theta}+3 H \Pi_{\Theta} & =-\frac{\partial \mathcal{H}}{\partial \Theta} .
\end{aligned}
$$


In the spatially flat Randall-Sundrum cosmology the Hubble expansion rate $H$ is related to the Hamiltonian via the modified Friedmann equation [26]

$$
H \equiv \frac{\dot{a}}{a}=\sqrt{\frac{8 \pi G}{3} \mathcal{H}\left(1+\frac{2 \pi G}{3 k^{2}} \mathcal{H}\right)}
$$

In addition, we will make use of the energy-momentum conservation equation

$$
\dot{\mathcal{H}}+3 H(\mathcal{H}+\mathcal{L})=0
$$

which, combined with the time derivative of (19), yields the second Friedmann equation in the form

$$
\dot{H}=-4 \pi G(\mathcal{H}+\mathcal{L})\left(1+\frac{4 \pi G}{3 k^{2}} \mathcal{H}\right)
$$

Thus, the Friedman equations are modified in the RSII cosmology. As far as we know the effects of these modifications on tachyon inflation were first studied by Bento, Bertolami and Sen [27].

To solve the system of equations (15)-(19), it is convenient to rescale the time as $t=\tau / k$ and express the system in terms of dimensionless quantities. Besides, we can eliminate the coupling constant $\lambda$ from the equations by appropriately rescaling the fields $\Phi$ and $\Theta$ and their conjugate fields $\Pi_{\Phi}$ and $\Pi_{\Theta}$. To this end we introduce the dimensionless functions

$$
\left.h=H / k, \quad \phi=\Phi /(k \sqrt{\lambda}), \quad \pi_{\phi}=\Pi_{\Phi} /\left(k^{2} \sqrt{\lambda}\right)\right), \quad \theta=k \Theta, \quad \pi_{\theta}=\Pi_{\Theta} /\left(k^{4} \lambda\right),
$$

and rescale the Lagrangian and Hamiltonian to obtain the dimensionless pressure and energy density:

$$
\begin{gathered}
\bar{p} \equiv \frac{\mathcal{L}}{k^{4} \lambda}=\frac{1}{2} \pi_{\phi}^{2}-\frac{\psi^{2}}{\theta^{4}} \frac{1}{\sqrt{1+\theta^{8} \pi_{\theta}^{2} / \psi}}, \\
\bar{\rho} \equiv \frac{\mathcal{H}}{k^{4} \lambda}=\frac{1}{2} \pi_{\phi}^{2}+\frac{\psi^{2}}{\theta^{4}} \sqrt{1+\theta^{8} \pi_{\theta}^{2} / \psi} .
\end{gathered}
$$

Following Steer and Vernizzi [1] we also introduce a combined dimensionless coupling

$$
\kappa^{2}=8 \pi \lambda G k^{2}
$$

Then, from (15)-(19) we obtain the following set of equations

$$
\begin{gathered}
\dot{\phi}=\pi_{\phi}, \\
\dot{\theta}=\frac{\theta^{4} \psi \pi_{\theta}}{\sqrt{1+\theta^{8} \pi_{\theta}^{2} / \psi}}, \\
\dot{\pi}_{\phi}=-3 h \pi_{\phi}-\frac{\psi}{2 \theta^{2}} \frac{4+3 \theta^{8} \pi_{\theta}^{2} / \psi}{\sqrt{1+\theta^{8} \pi_{\theta}^{2} / \psi}} \eta^{\prime} \\
\dot{\pi}_{\theta}=-3 h \pi_{\theta}+\frac{\psi}{\theta^{5}} \frac{4-3 \theta^{10} \eta \pi_{\theta}^{2} / \psi}{\sqrt{1+\theta^{8} \pi_{\theta}^{2} / \psi}}
\end{gathered}
$$


where

$$
\begin{gathered}
h \equiv \frac{\dot{a}}{a}=\sqrt{\frac{\kappa^{2}}{3} \bar{\rho}\left(1+\frac{\kappa^{2}}{12} \bar{\rho}\right)} \\
\psi=1+\theta^{2} \eta \\
\eta=\sinh ^{2}\left(\sqrt{\left.\frac{\kappa^{2}}{6} \phi\right),}\right. \\
\eta^{\prime}=\frac{d \eta}{d \phi}=\sqrt{\frac{\kappa^{2}}{6}} \sinh \left(\sqrt{\frac{2 \kappa^{2}}{3}} \phi\right) .
\end{gathered}
$$

In addition to Eqs. (26)-(30) we can solve in parallel the second Friedman equation

$$
\dot{h}=-\frac{\kappa^{2}}{2}(\bar{\rho}+\bar{p})\left(1+\frac{\kappa^{2}}{6} \bar{\rho}\right),
$$

and

$$
\dot{N}=h
$$

where $N$ is the number of e-folds as a function of $\tau$.

In Eqs. (26) $-(35)$ and from now on the overdot denotes a derivative with respect to $\tau$. Obviously, the explicit dependence on $\lambda$ in Eqs. (26)-(33) is eliminated and the only remaining free parameter is $\kappa$. Equations (26) and (27) can be used to express the pressure and energy density in terms of $\dot{\phi}$ and $\dot{\theta}$. One thus finds

$$
\begin{aligned}
& \bar{p}=\frac{1}{2} \dot{\phi}^{2}-\frac{\psi^{2}}{\theta^{4}} \sqrt{1-\dot{\theta}^{2} / \psi^{3}}, \\
& \bar{\rho}=\frac{1}{2} \dot{\phi}^{2}+\frac{\psi^{2}}{\theta^{4}} \frac{1}{\sqrt{1-\dot{\theta}^{2} / \psi^{3}}} .
\end{aligned}
$$

The functional dependence of $h$ on $\tau$ will be used to calculate the slow-roll parameters and the number of e-folds. The slow-roll parameters are defined as [11, 28]

$$
\epsilon_{i} \equiv \frac{d \ln \left|\epsilon_{i-1}\right|}{H d t}, \quad i \geq 1
$$

where

$$
\epsilon_{0} \equiv \frac{H_{*}}{H}
$$

and $H_{*}$ is the Hubble rate at an arbitrarily chosen time. Using the previously defined dimensionless Hubble rate $h$, the first two parameters can be written as

$$
\begin{gathered}
\epsilon_{1}=-\frac{\dot{h}}{h^{2}}, \\
\epsilon_{2}=2 \epsilon_{1}+\frac{\ddot{h}}{h \dot{h}} .
\end{gathered}
$$


The conditions for a slow-roll regime are satisfied when $\epsilon_{1}<1$ and $\epsilon_{2}<1$, and inflation ends when any of them exceeds unity. The effect of the radion and the tachyon can be seen if we compare the slow-roll parameters for the full model with those for the model with inverse quartic tachyon potential. The number of e-folds is defined through Eq. (35) as

$$
N=\int_{\tau_{\mathrm{i}}}^{\tau_{\mathrm{f}}} d \tau h,
$$

where the subscripts $\mathrm{i}$ and $\mathrm{f}$ denote the beginning and the end of inflation, respectively.

The slow-roll parameters are related to observable quantities, in particular to the tensorto-scalar ratio $r$ and the scalar spectral index $n_{\mathrm{s}}$ defined by

$$
\begin{gathered}
r=\frac{\mathcal{P}_{\mathrm{T}}}{\mathcal{P}_{\mathrm{S}}}, \\
n_{\mathrm{s}}=\frac{d \ln \mathcal{P}_{\mathrm{S}}}{d \ln q},
\end{gathered}
$$

where $\mathcal{P}_{\mathrm{S}}$ and $\mathcal{P}_{\mathrm{T}}$ are the power spectra of scalar and tensor perturbations, respectively, evaluated at the horizon, i.e., for a wave-number satisfying $q=a H$. Calculation of the spectra proceeds by identifying the proper canonical field and imposing quantization of the quadratic action for the near free field. The procedure for a general k-inflation is described in [29] and applied to the tachyon fluid in Refs. [11, 30]. It turns out that at the lowest order in $\epsilon_{1}$ and $\epsilon_{2}$, the power spectra may be expressed in the same way as in the standard tachyon inflation [1]:

$$
\begin{gathered}
\mathcal{P}_{\mathrm{T}} \simeq\left[1-2(1+C) \epsilon_{1}\right] \frac{16 G H^{2}}{\pi} \\
\mathcal{P}_{\mathrm{S}} \simeq\left[1-2(1+C-\alpha) \epsilon_{1}-C \epsilon_{2}\right] \frac{G H^{2}}{\pi \epsilon_{1}},
\end{gathered}
$$

where $C=-2+\ln 2+\gamma \simeq-0.72$, and $\alpha$ is a parameter related to the speed of sound expanded in $\epsilon_{1}$ :

$$
c_{\mathrm{s}}=1-2 \alpha \epsilon_{1}+O\left(\epsilon_{1}^{2}\right) .
$$

The parameter $\alpha$ will be calculated in the next section using the relation between $\epsilon_{1}$ and the speed of sound in the slow-roll approximation.

\section{Conditions for tachyon inflation}

\subsection{Inverse quartic potential}

To solve the system of equations (26)-(29) we need to choose initial conditions relevant for inflation. To this end we first solve a simpler case in which the radion is absent. The model is described by the pure tachyon Lagrangian with the inverse quartic potential [31]

$$
\mathcal{L}=-\frac{\lambda}{\Theta^{4}} \sqrt{1-g^{\mu \nu} \Theta_{, \mu} \Theta_{, \nu}}
$$


with the corresponding Hamiltonian

$$
\mathcal{H}=\frac{\lambda}{\Theta^{4}} \sqrt{1+\Pi_{\Theta}^{2} \Theta^{8} /\left(\lambda^{2}\right)} .
$$

As before, we rescale the time as $t=\tau / k$, absorb the coupling constant $\lambda$ into the conjugate field $\Pi_{\Theta}$ and introduce the dimensionless functions as in (22). Then, using (16), (18) and (49) we obtain the Hamilton equations in the form

$$
\begin{gathered}
\dot{\theta}=\frac{\theta^{4} \pi_{\theta}}{\sqrt{1+\theta^{8} \pi_{\theta}^{2}}} \\
\dot{\pi}_{\theta}=-3 h \pi_{\theta}+\frac{4}{\theta^{5} \sqrt{1+\theta^{8} \pi_{\theta}^{2}}}
\end{gathered}
$$

The dimensionless pressure and energy density are given by

$$
\begin{gathered}
\bar{p}=-\frac{1}{\theta^{4} \sqrt{1+\theta^{8} \pi_{\theta}^{2}}}=-\frac{1}{\theta^{4}} \sqrt{1-\dot{\theta}^{2}}, \\
\bar{\rho}=\frac{1}{\theta^{4}} \sqrt{1+\theta^{8} \pi_{\theta}^{2}}=\frac{1}{\theta^{4}} \frac{1}{\sqrt{1-\dot{\theta}^{2}}},
\end{gathered}
$$

Using this we also find a simple expression for the sound speed

$$
\left.c_{\mathrm{s}}^{2} \equiv \frac{\partial p}{\partial \rho}\right|_{\theta}=\frac{1}{1+\theta^{8} \pi_{\theta}^{2}}=1-\dot{\theta}^{2} .
$$

The Friedmann equations are given by Eq. (30) and (34) with (52) and (53). Hence, as before, the coupling $\lambda$ drops out and the only remaining parameter is $\kappa$ defined in (25). The system of equations (50)-(51) can be solved numerically for a chosen set of initial conditions. Once the solution for $H$ is found the slow-roll parameters can be easily calculated.

\subsection{Slow-roll approximation and initial conditions}

To find appropriate initial value of the field $\theta$, we first consider the pure tachyon model in the slow-roll approximation. Tachyon inflation is based upon the slow evolution of $\theta$ with the slow-roll conditions [11]

$$
\dot{\theta} \simeq \theta^{4} \pi_{\theta} \ll 1, \quad \dot{\pi}_{\theta} \ll 3 h \pi_{\theta},
$$

so that in the slow-roll approximation we may neglect the factors $\left(1-\dot{\theta}^{2}\right)^{1 / 2}$. Then, during inflation we have

$$
\begin{gathered}
h \simeq \frac{\kappa}{\sqrt{3} \theta^{2}}\left(1+\frac{\kappa^{2}}{12 \theta^{4}}\right)^{1 / 2}, \\
\dot{\theta} \simeq \frac{4}{3 h \theta} \simeq \frac{4 \theta}{\sqrt{3} \kappa}\left(1+\frac{\kappa^{2}}{12 \theta^{4}}\right)^{-1 / 2},
\end{gathered}
$$




$$
\ddot{\theta} \simeq \frac{4 \dot{\theta}}{\sqrt{3} \kappa}\left(1+\frac{\kappa^{2}}{12 \theta^{4}}\right)^{-3 / 2}\left(1+\frac{\kappa^{2}}{4 \theta^{4}}\right),
$$

and using (34) we also find

$$
\dot{h}=-\frac{\kappa^{2} \dot{\theta}^{2}}{2 \theta^{4}}\left(1+\frac{\kappa^{2}}{6 \theta^{4}}\right) .
$$

As a consequence, the slow-roll parameters (40) and (41) can be approximated by

$$
\begin{gathered}
\epsilon_{1} \simeq \frac{3}{2} \dot{\theta}^{2}\left(1+\frac{\kappa^{2}}{6 \theta^{4}}\right)\left(1+\frac{\kappa^{2}}{12 \theta^{4}}\right)^{-1} \\
\simeq \frac{8 \theta^{2}}{\kappa^{2}}\left(1+\frac{\kappa^{2}}{6 \theta^{4}}\right)\left(1+\frac{\kappa^{2}}{12 \theta^{4}}\right)^{-2}, \\
\epsilon_{2} \simeq 2 \frac{\ddot{\theta}}{h \dot{\theta}}-\dot{\theta}^{2} \frac{\kappa^{2}}{4 \theta^{4}}\left(1+\frac{\kappa^{2}}{6 \theta^{4}}\right)^{-1}\left(1+\frac{\kappa^{2}}{12 \theta^{4}}\right)^{-1} \\
\simeq \frac{8 \theta^{2}}{\kappa^{2}}\left(1+\frac{\kappa^{2}}{12 \theta^{4}}\right)^{-2}\left[1+\frac{\kappa^{2}}{4 \theta^{4}}-\frac{\kappa^{2}}{6 \theta^{4}}\left(1+\frac{\kappa^{2}}{6 \theta^{4}}\right)^{-1}\right] .
\end{gathered}
$$

In the slow roll regime we have $\kappa^{2} / \theta^{4} \gg 1$, so the corrections due to the RSII modification in Eqs. (56)-(61) will dominate over unity and we find

$$
\begin{aligned}
h & \simeq \frac{1}{6} \frac{\kappa^{2}}{\theta^{4}}, \quad \dot{\theta} \simeq 8 \frac{\theta^{3}}{\kappa^{2}}, \quad \ddot{\theta} \simeq 24 \frac{\theta^{2}}{\kappa^{2}} \dot{\theta}, \\
\epsilon_{1} & \simeq 3 \dot{\theta}^{2} \simeq 192 \frac{\theta^{6}}{\kappa^{4}}, \quad \epsilon_{2} \simeq 288 \frac{\theta^{6}}{\kappa^{4}} \simeq \frac{3}{2} \epsilon_{1} .
\end{aligned}
$$

In contrast, if we disregarded the RSII corrections we would obtain the usual slow-roll equations of tachyon inflation [11, 12, 13, 32] for the potential $V=\lambda / \theta^{4}$

$$
\begin{gathered}
h \simeq \frac{1}{\sqrt{3}} \frac{\kappa}{\theta^{2}}, \quad \dot{\theta} \simeq \frac{4}{\sqrt{3}} \frac{\theta}{\kappa}, \quad \ddot{\theta} \simeq \frac{4}{\sqrt{3}} \frac{\dot{\theta}}{\kappa}, \\
\epsilon_{1} \simeq \frac{3}{2} \dot{\theta}^{2} \simeq 8 \frac{\theta^{2}}{\kappa^{2}}, \quad \epsilon_{2} \simeq \epsilon_{1} .
\end{gathered}
$$

Hence, in the slow-roll regime the tachyon inflation in the RSII modified cosmology proceeds in a quite distinct way compared with that in the standard FRW cosmology. However, close to and at the end of inflation we have $\kappa^{2} / \theta_{\mathrm{f}}^{4} \ll 1$ and we can neglect the RSII cosmology corrections. Hence, the expressions (64) and (65) can be used at the end of inflation where we find

$$
\epsilon_{1}\left(\theta_{\mathrm{f}}\right) \simeq \epsilon_{2}\left(\theta_{\mathrm{f}}\right) \simeq \frac{8 \theta_{\mathrm{f}}^{2}}{\kappa^{2}} \simeq 1
$$

and

$$
h\left(\theta_{\mathrm{f}}\right) \simeq \frac{8}{\sqrt{3} \kappa} .
$$


In the slow-roll approximation the number of e-folds is given by

$$
N \simeq \frac{\kappa^{2}}{4} \int_{\theta_{0}}^{\theta_{\mathrm{f}}} \frac{d \theta}{\theta^{3}}\left(1+\frac{\kappa^{2}}{12 \theta^{4}}\right) \simeq \frac{\kappa^{2}}{8 \theta_{0}^{2}}\left(1+\frac{\kappa^{2}}{36 \theta_{0}^{4}}\right)-1 \simeq \frac{2}{3} \frac{1}{\epsilon_{1}\left(\theta_{0}\right)}-1
$$

where we have exploited $\kappa^{2} /\left(36 \theta_{0}^{4}\right) \gg 1$ at the beginning and $\kappa^{2} /\left(36 \theta_{\mathrm{f}}^{4}\right) \ll 1$ at the end of inflation together with the condition (66) . For comparison, in the standard tachyon inflation described by (64) and (65) we would obtain

$$
N_{\text {st.tach }} \simeq \frac{\kappa^{2}}{8 \theta_{0}^{2}}-1 \simeq \frac{1}{\epsilon_{1}\left(\theta_{0}\right)}-1
$$

For example, the choice $\kappa^{2}=5$ and $\theta_{0}=0.25$ leads to $N_{\text {st.tach }} \simeq 9$ whereas in RSII cosmology with the same parameters one finds $N \simeq 365$.

At this point it is convenient to estimate a phenomenologically acceptable range of the couplings $\lambda$ and $\kappa$. Although the evolution equations do not depend on $\lambda$, its approximate value is needed for choosing appropriate initial conditions for the radion field. The value of $\lambda$ may be estimated using the observational constraint on the amplitude of scalar perturbations. The approximate expression

$$
\mathcal{P}_{\mathrm{S}} \simeq \frac{G H^{2}}{\pi \epsilon_{1}}
$$

which follows from (46), is to be compared with the power spectrum amplitude $A_{s}$ measured by Planck [15]:

$$
A_{s} \simeq 2.2 \times 10^{-9}
$$

Hence, we must make sure that the condition

$$
\frac{H}{M_{\mathrm{P}}} \lesssim \sqrt{\pi A_{s}} \simeq 8.31 \times 10^{-5}
$$

is satisfied close to and at the end of inflation (where $\epsilon_{1} \lesssim 1$ ). (See also Ref. [33].) Here we define the Planck mass as $M_{\mathrm{P}}=G^{-1 / 2}$. According to Eq. (67) in the slow-roll approximation near the end of inflation we have

$$
\frac{H}{M_{\mathrm{P}}} \simeq \frac{8}{\sqrt{3}} \frac{k}{\kappa M_{\mathrm{P}}}=\sqrt{\frac{8}{3 \pi}} \frac{k^{2}}{\sqrt{\sigma}}
$$

yielding

$$
\frac{k}{\sigma^{1 / 4}} \lesssim 10^{-2}
$$

or

$$
\lambda \gtrsim 10^{8}
$$

To estimate $\kappa$ note first that the tension of a $\mathrm{D} p$-brane is given by 34

$$
\sigma=\frac{1}{(2 \pi)^{p} \alpha^{\prime(p+1) / 2} g_{\mathrm{s}}}
$$


where $g_{\mathrm{s}}$ is the string coupling constant and $1 /\left(2 \pi \alpha^{\prime}\right)$ is the string tension. Using this for $p=3$ from (74) we find a constraint

$$
g_{\mathrm{s}} \lesssim 4 \times 10^{-11}\left(\frac{M_{\mathrm{s}}}{k}\right)^{4}
$$

where $M_{\mathrm{s}}=1 / \sqrt{\alpha^{\prime}}$. Using this constraint we can choose $k$ and $M_{\mathrm{s}}$ such that the scale hierarchy

$$
H<k<M_{\mathrm{s}}<M_{\mathrm{P}}
$$

is satisfied. With this in mind we can give an order of magnitude estimate for an acceptable range of values of our free parameter $\kappa$. Requiring $k \gtrsim H$, from (73) it follows

$$
\kappa \gtrsim 8 / \sqrt{3}
$$

so we can safely choose $\kappa$ to vary in the range $1<\kappa<20$.

Equations (63) and (68) can be used to estimate the value of the ratio $\kappa^{2} / \theta_{0}^{3}$ by fitting the model to the observational parameters $r$ and $n_{\mathrm{s}}$ defined by (43) and (44). First, using (54) and (63) we can express the sound speed in terms of $\epsilon_{1}$ :

$$
c_{s} \simeq 1-\frac{1}{6} \epsilon_{1}
$$

and comparing this with (47) we find $\alpha=1 / 12$. In contrast, in the standard tachyon inflation the value $\alpha=1 / 6$ is obtained [11]. Using (45) and (46) we find up to the second order

$$
\begin{gathered}
r=16 \epsilon_{1}\left[1-\frac{1}{6} \epsilon_{1}+C \epsilon_{2}\right], \\
n_{\mathrm{s}}=1-2 \epsilon_{1}-\epsilon_{2}-\left[2 \epsilon_{1}^{2}+\left(2 C+\frac{17}{6}\right) \epsilon_{1} \epsilon_{2}+C \epsilon_{2} \epsilon_{3}\right],
\end{gathered}
$$

where it is understood that $\epsilon_{1}$ and $\epsilon_{2}$ take their values at or close to the beginning of inflation. Using these equations at linear order with $\epsilon_{2} \simeq 3 \epsilon_{1} / 2$ and (68), we find the approximate relations

$$
\begin{gathered}
r=\frac{32}{3} \frac{1}{N+1}, \\
n_{\mathrm{s}}=1-\frac{7}{3} \frac{1}{N+1}, \\
r=\frac{32}{7}\left(1-n_{\mathrm{s}}\right) .
\end{gathered}
$$

Had we used the usual relation $\epsilon_{2} \simeq \epsilon_{1}$ and (69) we would have obtained the standard tachyon-inflation relations [11, 13]

$$
\begin{gathered}
r=\frac{16}{N+1}, \\
n_{\mathrm{s}}=1-\frac{3}{N+1},
\end{gathered}
$$




$$
r=\frac{16}{3}\left(1-n_{\mathrm{s}}\right)
$$

Comparing $r$ and $n_{\mathrm{s}}$ with the latest observations we can fix the parameters $\epsilon_{1}, \epsilon_{2}$, and $N$. Then, from (68) we can determine the ratio $\kappa^{2} / \theta_{0}^{3}$ and for a chosen set of values of $\kappa$ we find the corresponding $\theta_{0}$. These values of $\kappa$ and the corresponding initial values $\theta_{0}$ as initial conditions are then used to solve the system of equation (50)-(51) for the pure tachyon and (26)-(29) for the tachyon-radion system.

There is no a priori reason to restrict possible initial values of the radion field $\Phi$ so we can choose a range of initial values based on the natural scale dictated by observations, i.e., the scale between $H$ and $M_{\mathrm{P}}$. Hence, a natural initial value for $\Phi$ would be of the order of $k$ or a few orders larger, say in the range 10 to $1000 k$. However, according to (22) the dimensionless radion field $\phi$ is rescaled with respect to $\Phi / k$ by a factor of $1 / \sqrt{\lambda} \simeq 10^{-4}$ so we can choose the initial value $\phi_{0}=\phi(0)$ in the range from 0.001 to 0.5 .

As we have mentioned in Introduction our tachyon model suffers from the so called reheating problem [7]. A possible way out is provided by string theory. String theory D-branes couple to the (pull-back of) antisymmetric tensor field $\mathcal{B}$ that combines the KalbRamond and electromagnetic fields. In this way there exist a natural coupling between the tachyon and the electromagnetic field. Therefore, this interaction could serve as a possible reheating mechanism at the end of inflation. In a tachyon model based on brane-antibrane annihilation resulting in a time dependent tachyon condensate it has been shown [10] that a coupling of massless fields to the time dependent tachyon condensate could yield a reheating efficient enough to overcome the above mention problem of a CDM dominance.

Another possible way out of the reheating problem could be the so called warm inflation [35]. Warm inflation is an alternative inflation scenario with no need for a reheating period. In warm inflation, dissipative effects are included during inflation, so that radiation is produced in parallel with the inflationary expansion and inflation ends when the universe heats up to become radiation dominated. This scenario has been successfully applied to tachyon inflation models [36, 37] and, in principle, should also work for our model. This requires further investigation which goes beyond the scope of the present paper.

\section{Numerical results}

The system of equations (26)-(29) is evolved numerically starting from $\tau=0$ up to some large $\tau$ of the order of 100 . The initial values $\theta_{0}$ and $\phi_{0}$ are chosen as described in the previous section and the initial conjugate momenta are taken to be $\pi_{\theta 0}=\pi_{\phi 0}=0$. The function $N(\tau)$ is solved simultaneously using (35) with $N(0)=0$. The time evolution of the slow roll parameters $\epsilon_{1}$ and $\epsilon_{2}$ are obtained using (401) and (41). The inflation ends at a point $\tau_{\mathrm{f}}$ at which $\epsilon_{1}\left(\tau_{\mathrm{f}}\right)=1$. The beginning of inflation at $\tau_{i}$ is then found by requiring $N\left(\tau_{\mathrm{f}}\right)-N\left(\tau_{\mathrm{i}}\right)=N$. The results for $\kappa=2, \theta_{0}=0.25$ and $\phi_{0}=0.4$ are presented in Fig. 1 together with the results calculated for the system (50)-(51) within the standard FRW cosmology with the same initial $\theta_{0}=0.25$ and $\pi_{\theta 0}=0$.

To calculate the quantities $n_{s}$ and $r$ we proceed as follows. For a chosen pair of $(N, \kappa)$ we first find the initial value $\theta_{0}$ from Eq. (68). Then, for a chosen set of initial values $\phi_{0}$, $\pi_{\theta 0}, \pi_{\phi 0}($ at $\tau=0)$ we find the corresponding $\phi_{\mathrm{i}}, \pi_{\theta \mathrm{i}}, \pi_{\phi \mathrm{i}}$ at $\tau=\tau_{\mathrm{i}}$ and calculate $h, \dot{h}$ and $\ddot{h}$ 

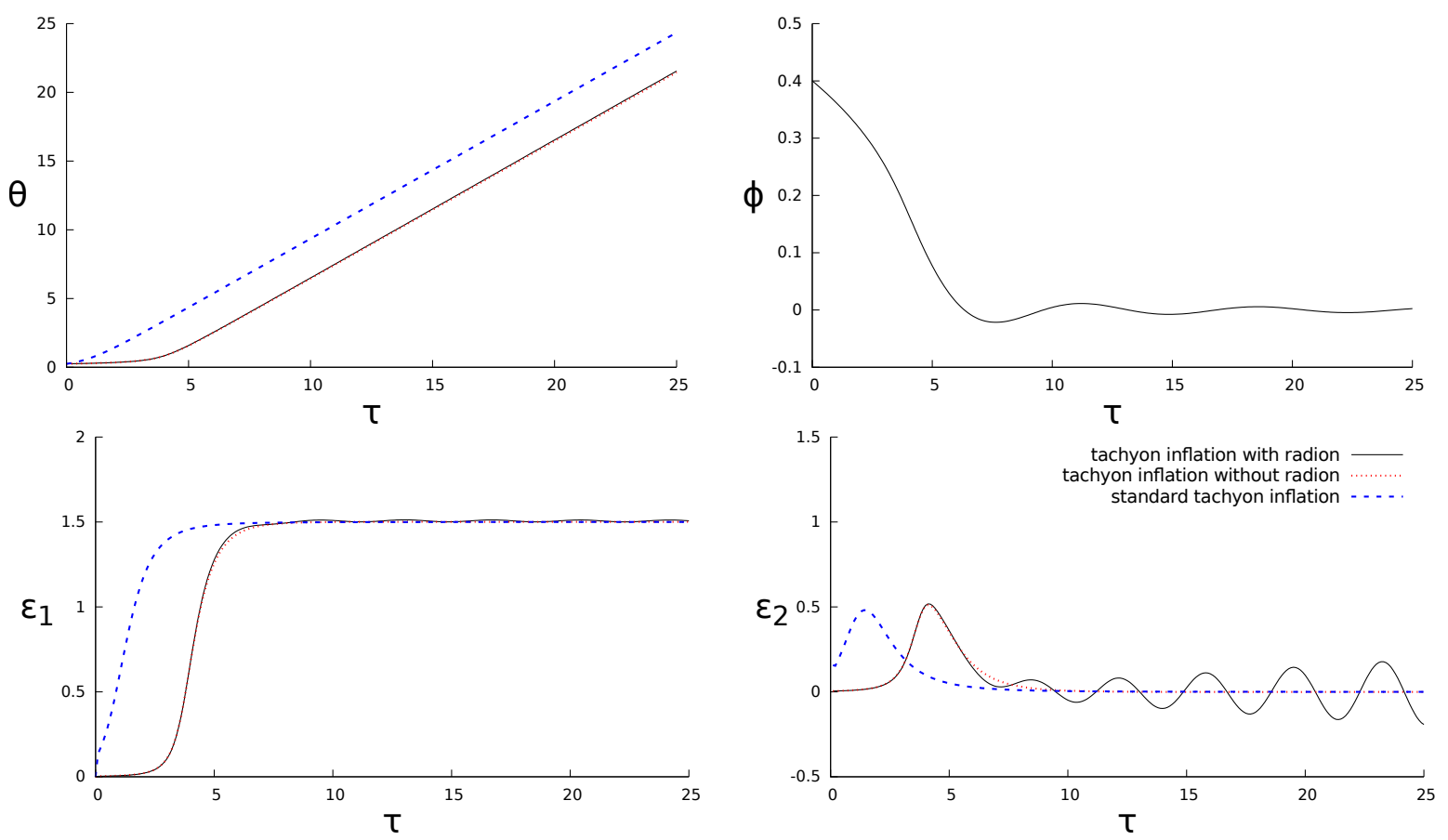

Figure 1: Time evolution of the tachyon $\theta$ (top left), the radion $\phi$ (top right) fields and of the slow-roll parameters $\epsilon_{1}$ (bottom left) and $\epsilon_{2}$ (bottom right) for $\kappa=2, \phi_{0}=0.4$, $\pi_{\theta 0}=\pi_{\phi 0}=0, \theta_{0}=0.25$, in the tachyon-inflation model with inverse quartic potential in the RSII cosmology with radion (full black line) and without radion (dotted red line). The dashed blue line represents the corresponding results for the tachyon-inflation model with inverse quartic potential in the standard FRW cosmology and no radion.

using equations (26) $-(\sqrt{30})$ with (23) and (24). From this we find $\epsilon_{1}\left(\theta_{\mathrm{i}}\right)$ and $\epsilon_{2}\left(\theta_{\mathrm{i}}\right)$ using the defining expressions (40) and (41) and calculate the parameters $r$ and $n_{\mathrm{s}}$ from (81) and (82), respectively. In Fig. 2 we present $n_{s}$ and $r$ as functions of $\kappa$ and $N$ for a fixed $\phi_{0}=0.01$.
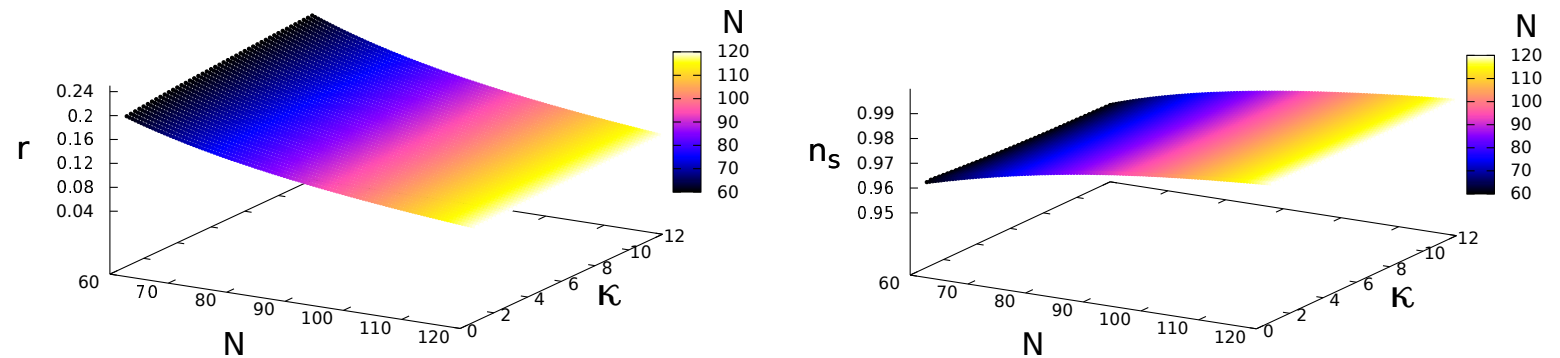

Figure 2: The quantities $r$ (left) and $n_{\mathrm{s}}$ (right) as functions of the parameters $N$ and $\kappa$ as indicated on the horizontal axes for initial $\phi_{0}=0.01$. The initial $\theta_{0}$ varies with $N$ and $\kappa$ according to Eq. (68).

In Fig. 3 we present the $n_{s}-r$ diagram with 10000 points superimposed on the observational constraints taken from the Planck Collaboration 2015 [15]. Each point in the diagram 
corresponds to a set $\left(N, \kappa, \phi_{0}\right)$ chosen randomly in the range $60 \leq N \leq 120,1 \leq \kappa \leq 12$ and $0 \leq \phi_{0} \leq 0.5$.

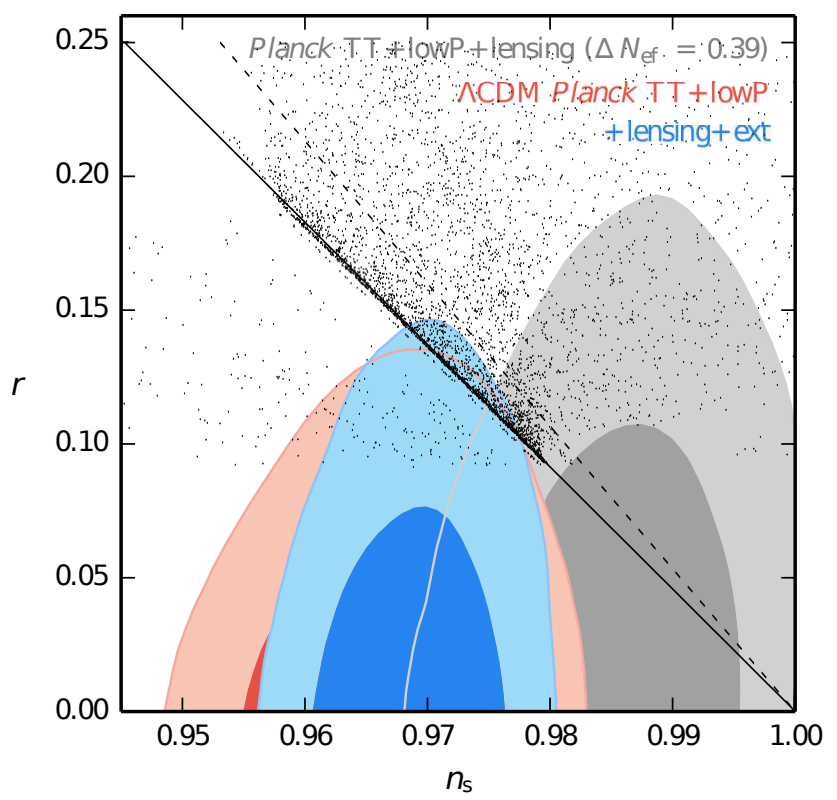

Figure 3: $r$ versus $n_{\mathrm{s}}$ diagram with observational constraints from Ref. [15]. The dots represent the calculation in the tachyon-radion model for various $N, \kappa$ and $\phi_{0}$ chosen randomly in the range $60 \leq N \leq 120,1 \leq \kappa \leq 12$ and $0 \leq \phi_{0} \leq 0.5$. The full line represents the slow-roll approximation (Eq. (85)) of the RSII model with no radion. The dashed line represents the slow-roll approximation (Eq. (88)) of the standard tachyon model with inverse quartic potential.

To obtain a more favorable distribution of points within the $2 \sigma$ area measured by the Planck collaboration we have used the distribution histograms of the number of e-folds $N$ and the parameters $\kappa$ and $\phi_{0}$. In this way we have been guided to restrict $N$ to range between 85 and 110, $\kappa$ between 1 and 8 , and $\phi_{0}$ between 0 and 0.5 . The outcome of these constraints is presented in Fig. 4. In this figure one notices basically two bands: the dominant one almost parallel to the $r$ axis with most data points concentrated close to the line corresponding to the RSII model prediction without radion and the less dens one almost parallel to the $n_{s}$ axis, with $r$ between 0.1 and 0.13 .

From a quite large data set of numerical results we find that smaller values of $\kappa$ give a better agreement with observational data from the Planck mission. For example, if we fix $\kappa=2$ and vary $N$ in a wider range $N 60 \leq N \leq 120)$ and $\phi_{0}$ in a bit narrower range $\left.0 \leq \phi_{0} \leq 0.25\right)$ we obtain an interesting splitting of points into three disconnected clusters (Fig. [5). This splitting is a clear manifestation of the nonlinearity of Eqs. (26)-(29). The most pronounced cluster of numerical points fits in quite well within the $2 \sigma$ regions of constraints given by the Planck collaboration.

A still better agreement between our model and the observational data constraints is obtained for a very narrow range of parameters as shown in Figs. 6 and 7 . The best fit is obtained for $\kappa=1.25, \phi_{0}=0.05$ and $115 \leq N \leq 120$. 


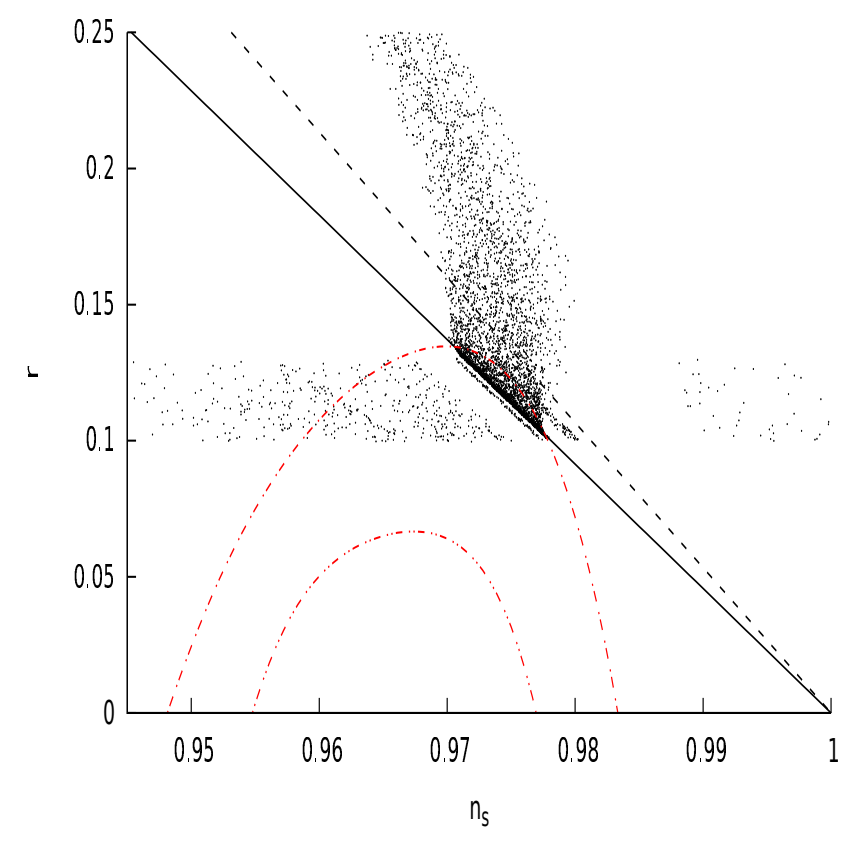

Figure 4: Same as in Fig. 3 for $N, \kappa$, and $\phi_{0}$ chosen randomly in the intervals $85 \leq N \leq 110$, $1 \leq \kappa \leq 8$ and $0 \leq \phi_{0} \leq 0.5$. The inner and outer dash-dotted lines denote the Planck contours of the one and two $\sigma$ constraints, respectively.

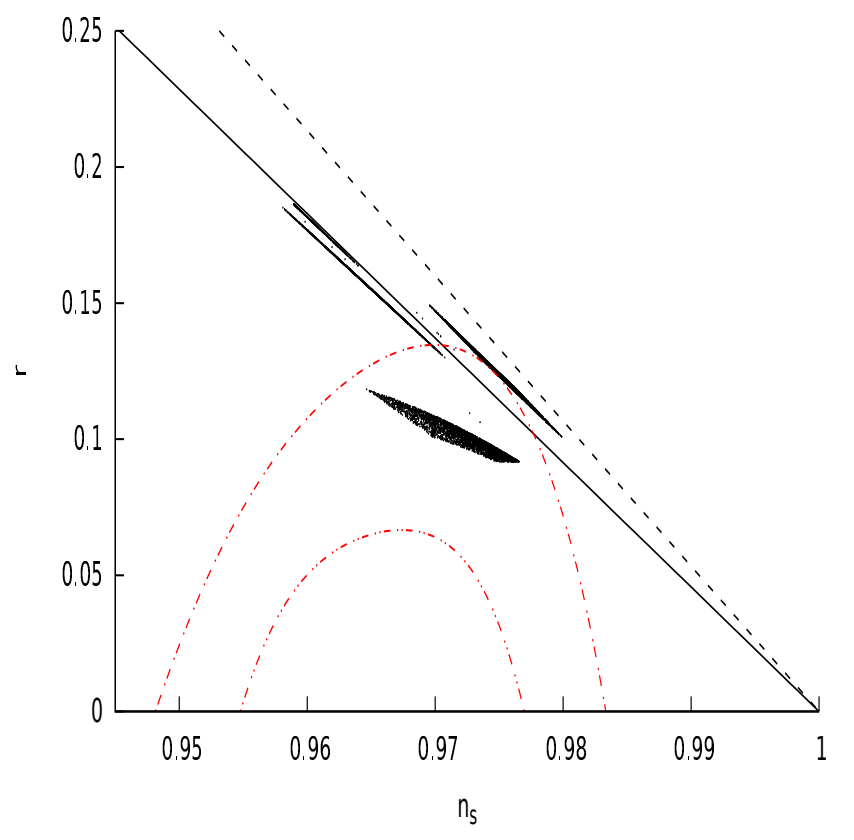

Figure 5: Same as in Fig. 4 for a fixed $\kappa=2$ and $N$ and $\phi_{0}$ chosen randomly in the intervals $60 \leq N \leq 120$ and $0 \leq \phi_{0} \leq 0.25$. 


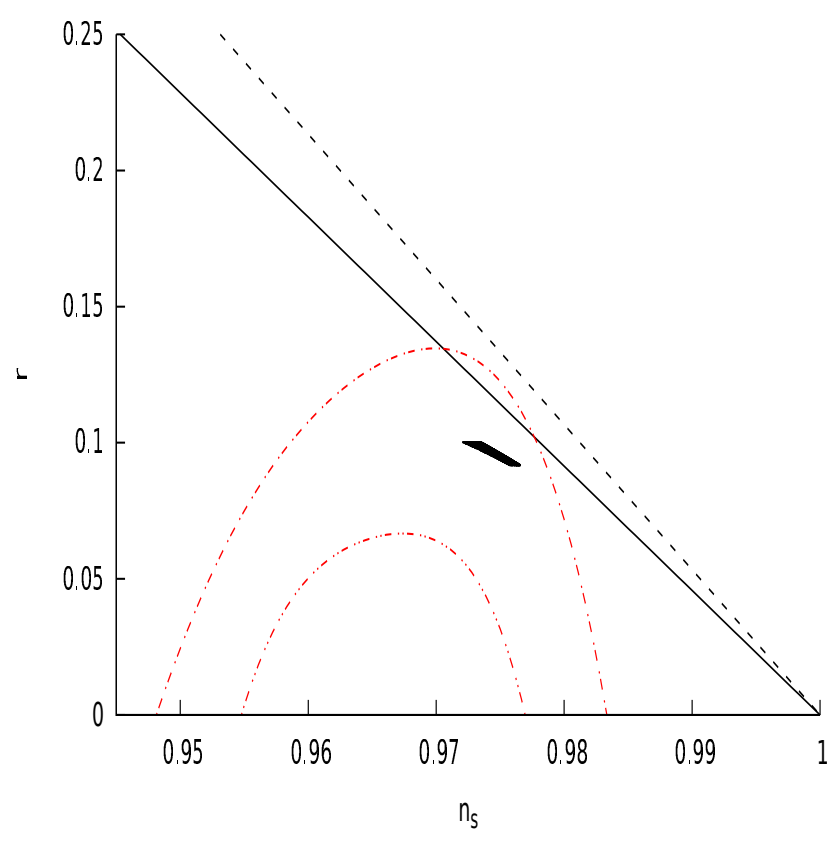

Figure 6: Same as in Fig. 5 for $N$ and $\phi_{0}$ chosen randomly in the intervals $110 \leq N \leq 120$ and $0.1 \leq \phi_{0} \leq 0.2$.

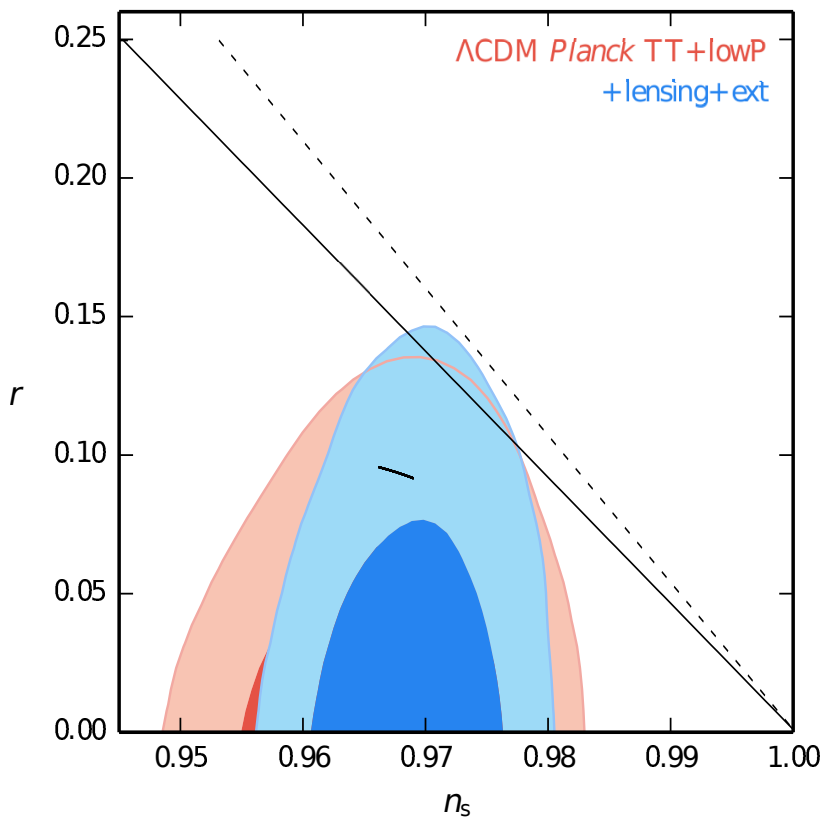

Figure 7: Same as in Fig. 3 for fixed $\kappa=1.25$ and $\phi_{0}=0.05$ and $N$ chosen randomly in the interval $115 \leq N \leq 120$.

\section{$5 \quad$ Summary and conclusions}

We have investigated a model of inflation based on the dynamics of a D3-brane in the $\mathrm{AdS}_{5}$ bulk of the RSII model. The bulk metric is extended to include the back reaction of the 
radion excitations.

We have shown that the slow-roll equations of the tachyon inflation are quite distinct to those of the standard tachyon inflation with the same potential. In particular, the departure of the sound speed from unity equals $c_{\mathrm{s}}-1 \simeq-\epsilon_{1} / 6$ in contrast to the standard result $c_{\mathrm{s}}-1 \simeq-\epsilon_{1} / 3$. The $n_{\mathrm{s}}-r$ relation in our model is substantially different from the standard one and is closer to the best observational value, as shown in Fig. 6. Note that the largest concentration of numerical results, represented by the points in the plot in Fig. 6, are within $2 \sigma$ of the Planck TT+lowP and Planck TT+lowP+lensing $+\mathrm{BAO}+\mathrm{JLA}+\mathrm{H}_{0}$ (red and light blue shaded regions, respectively). Clearly, the agreement with observations is not ideal and it is fair to say that the present model is disfavored but not excluded. However, one should bare in mind that the model is based on the brane dynamics which results in a definite potential with one free parameter only. In contrast, the majority of other tachyon potentials discussed in the literature are chosen arbitrarily and adjusted so that the results agree with observations.

In this work we have analyzed the simplest tachyon model that stems from the dynamics of a D3-brane in an $\mathrm{AdS}_{5}$ bulk yielding basically an inverse quartic potential. In principle, the same mechanism could lead to a more general tachyon potential if the $\mathrm{AdS}_{5}$ background metric is deformed by the presence of matter in the bulk, e.g., in the form of a minimally coupled scalar field with an arbitrary self-interaction potential. This will be the subject of our investigation in the future.

\section{Acknowledgments}

This work has been supported by ICTP - SEENET-MTP project PRJ-09 Cosmology and Strings. The work of N. Bilić has been supported by the Croatian Science Foundation under the project (IP-2014-09-9582). D. Dimitrijevic, G. Djordjevic and M. Milosevic acknowledge support provided by the Serbian Ministry for Education, Science and Technological Development under the projects No 176021 and No 174020. G. Djordjevic would like to thank CERN-Theoretical Physics Department for kind hospitality during the finalization of this paper.

\section{References}

[1] A. A. Starobinsky, JETP Lett. 30, 682 (1979) [Pisma Zh. Eksp. Teor. Fiz. 30, 719 (1979)].

[2] A. H. Guth, Phys. Rev. D 23, 347 (1981).

[3] A. D. Linde, Phys. Lett. B 116, 335 (1982). doi:10.1016/0370-2693(82)90293-3

[4] M. Fairbairn and M. H. G. Tytgat, Phys. Lett. B 546, 1 (2002) hep-th/0204070;

[5] A. Feinstein, Phys. Rev. D 66, 063511 (2002) doi:10.1103/PhysRevD.66.063511 hep-th/0204140.

[6] G. Shiu and I. Wasserman, Phys. Lett. B 541, 6 (2002) hep-th/0205003. 
[7] L. Kofman and A. D. Linde, JHEP 0207, 004 (2002) [hep-th/0205121].

[8] M. Sami, P. Chingangbam and T. Qureshi, Phys. Rev. D 66, 043530 (2002) hep-th/0205179].

[9] G. Shiu, S. H. Henry Tyee and I. Wasserman, Phys. Rev. D 67, 083517 (2003) [hep-th/0207119].

[10] J. M. Cline, H. Firouzjahi and P. Martineau, JHEP 0211, 041 (2002) hep-th/0207156.

[11] D. A. Steer and F. Vernizzi, Phys. Rev. D 70, 043527 (2004) hep-th/0310139].

[12] S. del Campo, R. Herrera and A. Toloza, Phys. Rev. D 79, 083507 (2009) arXiv:0904.1032 [astro-ph.CO]].

[13] S. Li and A. R. Liddle, JCAP 1403, 044 (2014) [arXiv:1311.4664 [astro-ph.CO]].

[14] G. W. Gibbons, Phys. Lett. B 537, 1 (2002); S. Mukohyama, Phys. Rev. D 66, 024009 (2002); M. Sami, Mod. Phys. Lett. A 18, 691 (2003); A. Mazumdar, S. Panda and A. Perez-Lorenzana, Nucl. Phys. B 614, 101 (2001); Y. S. Piao, R. G. Cai, X. Zhang and Y. Z. Zhang, Phys. Rev. D 66, 121301 (2002); C. j. Kim, H. B. Kim and Y. b. Kim, Phys. Lett. B 552, 111 (2003); T. Matsuda, Phys. Rev. D 67, 083519 (2003); Z. K. Guo, Y. S. Piao, R. G. Cai and Y. Z. Zhang, Phys. Rev. D 68, 043508 (2003); M. Majumdar and A. C. Davis, arXiv:hep-th/0304226; S. Nojiri and S. D. Odintsov, Phys. Lett. B 571, 1 (2003); V. Gorini, A. Y. Kamenshchik, U. Moschella and V. Pasquier, Phys. Rev. D 69, 123512 (2004); B. C. Paul and M. Sami, Phys. Rev. D 70, 027301 (2004); G. N. Felder and L. Kofman, Phys. Rev. D 70, 046004 (2004); J. M. Aguirregabiria and R. Lazkoz, Mod. Phys. Lett. A 19, 927 (2004); J. Raeymaekers, JHEP 0410, 057 (2004); N. Barnaby and J. M. Cline, arXiv:hep-th/0410030; Joris Raeymaekers, JHEP 0410, 057 (2004); K. L. Panigrahi, Phys. Lett. B601, 64 (2004); P. Chingangbam, S. Panda and A. Deshamukhya, JHEP 0502, 052 (2005) [hep-th/0411210].

[15] P. A. R. Ade et al. [Planck Collaboration], arXiv:1502.01589 [astro-ph.CO].

[16] D. Pirtskhalava, L. Santoni and E. Trincherini, JCAP 1606, no. 06, 051 (2016) arXiv:1511.01817 [hep-th]].

[17] L. Randall and R. Sundrum, Phys. Rev. Lett. 83, 4690 (1999)

[18] W. D. Goldberger and M. B. Wise, Phys. Rev. Lett. 83, 4922 (1999) hep-ph/9907447.

[19] J. E. Kim, G. B. Tupper, R. D. Viollier, Phys. Lett. B 593, 209 (2004).

[20] A. O. Barvinsky, Phys. Rev. D 65, 062003 (2002) hep-th/0107244].

[21] N. Bilic and G. B. Tupper, arXiv:1302.0955 [hep-th].

[22] N. Bilić and G. B. Tupper, Central Eur. J. Phys. 12, 147 (2014) arXiv:1309.6588 [hepth]]. 
[23] M. Bordemann and J. Hoppe, Phys. Lett. B 325359 (1994); N. Ogawa, Phys. Rev. D 62085023 (2000).

[24] R. Jackiw, (2002) Lectures on Fluid Mechanics (Springer Verlag, Berlin, 2002).

[25] L. R. W. Abramo and F. Finelli, Phys. Lett. B 575, 165 (2003) astro-ph/0307208].

[26] R. Maartens and K. Koyama, Living Rev. Rel. 13, 5 (2010) arXiv:1004.3962 [hep-th]].

[27] M. C. Bento, O. Bertolami and A. A. Sen, Phys. Rev. D 67, 063511 (2003);

[28] D. J. Schwarz, C. A. Terrero-Escalante and A. A. Garcia, Phys. Lett. B 517, 243 (2001) astro-ph/0106020.

[29] J. Garriga and V. F. Mukhanov, Phys. Lett. B 458, 219 (1999) doi:10.1016/S03702693(99)00602-4 [hep-th/9904176].

[30] A. V. Frolov, L. Kofman and A. A. Starobinsky, Phys. Lett. B 545, 8 (2002) doi:10.1016/S0370-2693(02)02582-0 [hep-th/0204187.

[31] N. Bilic, D.D. Dimitrijevic, G.S. Djordjevic, M. Milosevic, M. Stojanovic, AIP Conf. Proc. 1722, 050002 (2016); M. Milosevic, D.D. Dimitrijevic, G.S. Djordjevic, M.D. Stojanovic, Serb. Astron. J. 192, 1-8 (2016).

[32] M. R. Garousi, M. Sami and S. Tsujikawa, Phys. Rev. D 70, 043536 (2004) hep-th/0402075.

[33] S. Thomas and J. Ward, Phys. Rev. D 72, 083519 (2005) hep-th/0504226.

[34] C.V. Johnson, D-Branes (Cambridge University Press, Cambridge, 2003).

[35] A. Berera, Phys. Rev. Lett. 75, 3218 (1995), astro-ph/9509049]; A. Berera, Phys. Rev. D 54, 2519 (1996), hep-th/9601134.

[36] R. Herrera, S. del Campo and C. Campuzano, JCAP 0610, 009 (2006), astro-ph/0610339; S. del Campo, R. Herrera and J. Saavedra, Eur. Phys. J. C 59, 913 (2009) arXiv:0812.1081.

[37] M. R. Setare and V. Kamali, JHEP 1303, 066 (2013) arXiv:1302.0493]; A. Bhattacharjee and A. Deshamukhya, Mod. Phys. Lett. A 28, 1350036 (2013), arXiv:1302.1272]; X. M. Zhang and J. Y. Zhu, JCAP 1402, 005 (2014), arXiv:1311.5327]; M. R. Setare and V. Kamali, Phys. Lett. B 736, 86 (2014) arXiv:1407.2604]. A. Cid, Phys. Lett. B 743, 127 (2015) arXiv:1503.00714]; M. Motaharfar and H. R. Sepangi, arXiv:1604.00453; V. Kamali, S. Basilakos and A. Mehrabi, arXiv:1604.05434 [gr-qc]. 